\section{Design of a photonic band gap polarizer}

\author{
Ravindra K. Sinha, MEMBER SPIE, and \\ Yogita Kalra, MEMBER SPIE \\ University of Delhi, Faculty of Technology, Delhi College of \\ Engineering, Department of Applied Physics, TIFAC- \\ Center of Relevance and Excellence in Fiber Optics and \\ Optical Communications, Bawana Road, Delhi 110 \\ 042, India \\ E-mail: dr_rk_sinha@yahoo.com
}

Abstract. We explore the application of photonic band gaps (PBGs) in photonic crystal structures to propose the design of an ultracompact PBG polarizer. The existence of complete PBGs in certain photonic crystal structures and the variation introduced in the PBGs by the creation of defects has been utilized to design a PBG polarizer at $1.55 \mu \mathrm{m}$ with a degree of polarization equal to 1 leading to the formation of a super polarizer. () 2006 Society of Photo-Optical Instrumentation Engineers. [DOI: 10.1117/1.2372461]

Subject terms: photonic band gap; photonic crystals; polarizer.

Paper 060348LR received May 10, 2006; revised manuscript received Aug. 3, 2006; accepted for publication Aug. 24, 2006; published online Nov. 6, 2006.

\section{Introduction}

Photonic crystals (PhCs), also known as photonic band gap (PBG) structures, which have gained worldwide interest in the recent past, are periodic structures belonging to a new class of artificial materials that allow one to manipulate the flow of light. ${ }^{1-4}$ After the first proposal of PhCs by John and Yablonovitch, research and development in this field is taking place at a feverish pace because of the tremendous potential these structures hold. ${ }^{5-8}$ Because PBG structures allow strong control over the propagation of light, some of the most exciting applications of these structures are based on the functionalities through the incorporation of defects in periodic lattice leading to the design of $\mathrm{PhC}$ heterostructure-based PBG waveguides and devices. Defects influence the photonic band structure of the $\mathrm{PhC}$ and can result in the flow or confinement of light along particular pathways in the crystal. Moreover, PBGs in these structures are polarization sensitive.

These properties of PhC structures have been used to design various polarization sensitive devices, such as polarization splitters, multiplexers, demultiplexers, and twodimensional PhC lasers. ${ }^{9-14}$

Polarization discriminating optical elements are widely used in fiber optic applications and in quantum information processing. An important device in this class is the polarizer, which selectively attenuates light in one state of polarization while transmitting the orthogonal state of polarization.

In this letter, we envisage the existence of PBG as well as complete $\mathrm{PBG}(\mathrm{CPBG})$ and their polarization sensitivity to design PBG polarizer. Earlier polarizers that have been reported are based completely on the pseudo-band gaps exhibited by PhC structures. ${ }^{15,16}$ The PBG computations

0091-3286/2006/\$22.00 @ 2006 SPIE have been done using the plane wave expansion (PWE) method and the polarizer has been modeled using the finite difference time domain (FDTD) method.

\section{Design Parameters}

To design a PBG polarizer, we consider a PBG structure composed of a honeycomb lattice of $\mathrm{Si}(n=3.42)$ rods in air with lattice constant $a=0.885 \mu \mathrm{m}$. We first study the variation of complete PBG by varying the normalized rod radii $r / a$, where $r$ is the radius of the rods. We select a PBG structure composed of honeycomb lattice of Si rods in air with normalized rod radius $r / a=0.24$ to have a maximum range of CPBG. Figures 1(a) and 1(b) show the photonic band diagrams for transverse electric (TE) and transverse magnetic (TM) polarizations for this $\mathrm{PhC}$ structure, obtained using the PWE method. This PBG structure exhibits CPBG for normalized frequency range $0.53711 \leq a / \lambda$ $\leq 0.58793$.

Further, an input waveguide is formed by creating a linear waveguide by removing two rows of dielectric rods. Since the considered PBG structure possesses the CPBG, light for both TE and TM polarization in the wavelength range $1.51 \mu \mathrm{m} \leq \lambda \leq 1.65 \mu \mathrm{m}$ and hence both (TE and TM) polarization states can be guided in the input waveguide.

Further, to design a PBG polarizer, we have to design a $\mathrm{PhC}$ geometrical heterostructure in such a way that the light of one polarization is blocked while the light of another

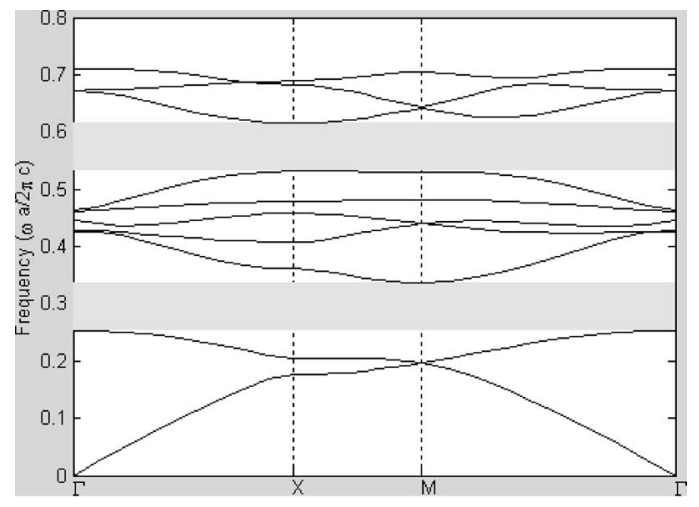

(a)

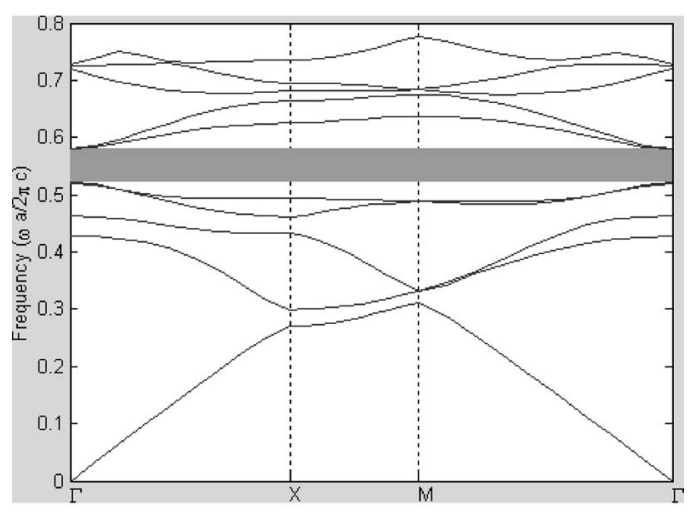

(b)

Fig. 1 Band diagram for the PBG structure composed of $\mathrm{Si}$ rods with $r / a=0.24$ in air in honeycomb lattice (a) for TM mode, and (b) for TE mode. 

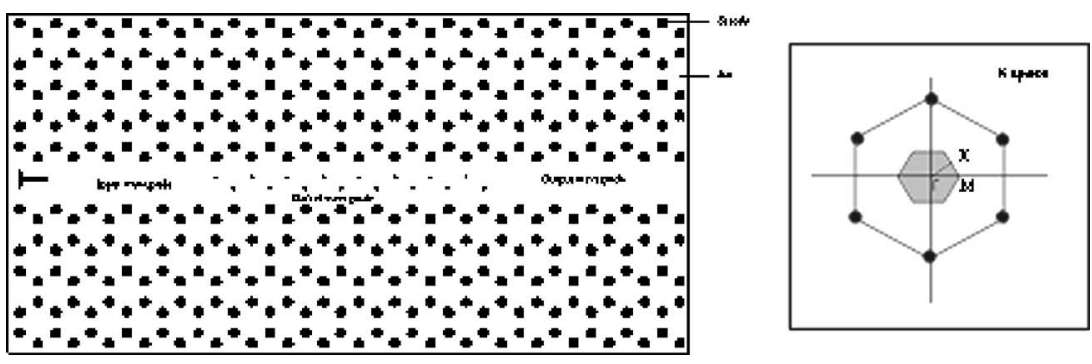

Fig. 2 Schematic view of the PBG polarizer.

polarization is allowed to pass, so that at the output end, the wavelength of one polarization is obtained. This property of the sensitivity of the PBGs to the polarization of light can be used to design a PBG polarizer. So after the input waveguide, modifications are made in the PBG structure such that it exhibits a band gap for either of the two polarizations, which overlaps with the CPBG regime.

To, obtain such a structure, we create a linear defect waveguide in the $\mathrm{PhC}$ structure after the input waveguide by changing the radius of the $\mathrm{Si}$ rods in the two rows, which is followed by an output waveguide formed by removing two rows of the $\mathrm{Si}$ rods. Figure 2 shows the schematic diagram of the PBG polarizer.

In order to find the parameters of the defect waveguide, we investigate the variation of PBGs by changing the radius of defect rods in the linear defect waveguide using the PWE method. Table 1 shows the range of the PBGs for the TE and TM polarizations by changing the radius of the defect rods.

To design a polarizer at $\lambda=1.55 \mu \mathrm{m}$, the radius of the defect rods in the linear defect waveguide is chosen to be $0.08 a$. This defect waveguide supports only TE modes but exhibits a PBG for TM mode in the range $0.56006 \leq a / \lambda$ $\leq 0.57243$ as evident from Table 1 providing a bandwidth of $34 \mathrm{~nm}$.

\section{Numerical Analysis}

The designed PBG polarizer has been modeled using the FDTD method. Now if at the input end, the light of both the TE and TM polarizations is launched in the input waveguide, then at the output end, the light of TE polarization is obtained as TE modes are allowed to propagate in the defect waveguide, whereas TM modes are not allowed and hence are reflected back as shown in Figs. 3(a) and 3(b).

The dimensions of the PBG polarizer lie in the micrometer range as in the present case, the length of the polarizer is $30 \mu \mathrm{m}$, which is evident from the snapshots in Figs. 3(a) and 3(b). As mentioned earlier, the designed PBG polarizer provides a bandwidth of $34 \mathrm{~nm}$, which is obtained from the pseudo-PBG introduced by making the defect waveguide.

The performance of a polarizer is conventionally characterized by the degree of polarization $P$ that is defined as

$P=\frac{\left|I_{T E}-I_{T M}\right|}{I_{T E}+I_{T M}}$,

where $I_{T E}\left(I_{T M}\right)$ is the intensity of the outgoing TE (TM) component, which is obtained as 1 as the TM mode is completely blocked by the defect waveguide in this case and hence leading to the design of a super polarizer.

The transmittance $T$ of a polarizer is defined here as the ratio of the intensity of the TE mode (in this case) passing through the polarizer $\left[I_{T E(o u t)}\right]$ to the incident intensity of the TE mode $\left[I_{T E(i n)}\right]$

$T=\frac{I_{T E(\text { out })}}{I_{T E(\text { in })}}$

and is obtained as 0.74 .

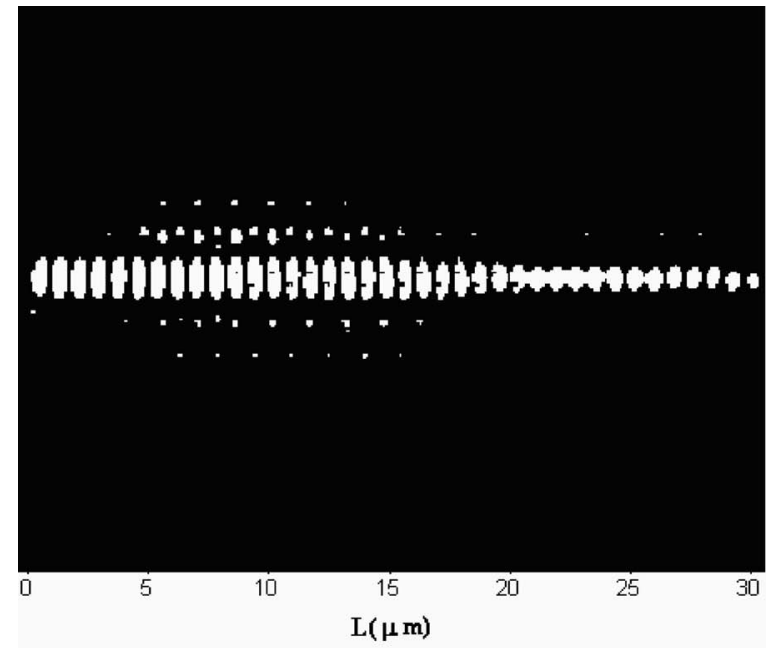

(a)

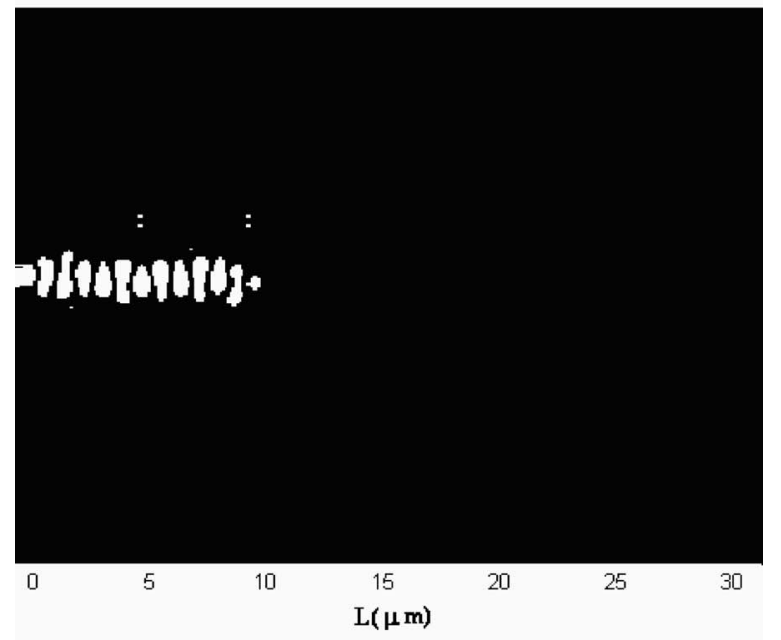

(b)

Fig. 3 Snapshot of the PBG polarizer at $1.55 \mu \mathrm{m}$ for TE mode (a) and (b) for TM mode. 
Table 1 PBGs for TM and TE polarizations with varying defect radii as calculated using the PWE method.

\begin{tabular}{|c|c|c|}
\hline $\begin{array}{l}\text { Defect } \\
\text { Radius } \\
\left(r_{d} / a\right)\end{array}$ & $\begin{array}{l}\text { Photonic Band Gap } \\
\text { Range for } \\
\text { TM Polarization }\end{array}$ & $\begin{array}{l}\text { Photonic Band Gap } \\
\text { Range for } \\
\text { TE Polarization }\end{array}$ \\
\hline 0.08 & $\begin{array}{l}0.54967 \leq a / \lambda \leq 0.55829 \\
0.56006 \leq a / \lambda \leq 0.57243\end{array}$ & \\
\hline 0.10 & $\begin{array}{c}0.54422 \leq a / \lambda \leq 0.5542 \\
0.55476 \leq a / \lambda \leq 0.57092\end{array}$ & $0.57101 \leq a / \lambda \leq 0.5542$ \\
\hline 0.12 & $\begin{array}{l}0.54209 \leq a / \lambda \leq 0.55157 \\
0.55238 \leq a / \lambda \leq 0.56914\end{array}$ & $0.56919 \leq a / \lambda \leq 0.58238$ \\
\hline 0.14 & $\begin{array}{l}0.54074 \leq a / \lambda \leq 0.54962 \\
0.55069 \leq a / \lambda \leq 0.56731\end{array}$ & $0.56723 \leq a / \lambda \leq 0.57824$ \\
\hline 0.16 & $\begin{array}{l}0.53893 \leq a / \lambda \leq 0.54684 \\
0.54785 \leq a / \lambda \leq 0.56151\end{array}$ & \\
\hline 0.18 & $0.55658 \leq a / \lambda \leq 0.56482$ & \\
\hline 0.20 & $0.54352 \leq a / \lambda \leq 0.5519$ & $0.56472 \leq a / \lambda \leq 0.57933$ \\
\hline 0.22 & $0.55597 \leq a / \lambda \leq 0.56092$ & $0.5649 \leq a / \lambda \leq 0.56686$ \\
\hline 0.26 & $\begin{array}{c}0.54065 \leq a / \lambda \leq 0.5472 \\
0.55604 \leq a / \lambda \leq 0.58878 \\
0.5889 \leq a / \lambda \leq 0.5942\end{array}$ & $0.55604 \leq a / \lambda \leq 0.58878$ \\
\hline 0.28 & $\begin{array}{l}0.53703 \leq a / \lambda \leq 0.54523 \\
0.55274 \leq a / \lambda \leq 0.58389\end{array}$ & $0.55274 \leq a / \lambda \leq 0.57606$ \\
\hline 0.30 & $\begin{array}{c}0.535 \leq a / \lambda \leq 0.54117 \\
0.54999 \leq a / \lambda \leq 0.56447\end{array}$ & $0.52651 \leq a / \lambda \leq 0.53445$ \\
\hline 0.32 & $0.5673 \leq a / \lambda \leq 0.57441$ & $0.59161 \leq a / \lambda \leq 0.59899$ \\
\hline
\end{tabular}

Similarly, by tailoring the radius of the Si rods in the $\mathrm{PhC}$ defect waveguide in $\mathrm{PhC}$, the PBG polarizer can be designed at the desired wavelength, operational range, and thereby the desired bandwidth.

\section{Conclusion}

We have proposed the design of the PBG polarizer by utilizing the PBGs exhibited by $\mathrm{PhC}$ structures, which have been modeled using the FDTD method. The dimension of the PBG polarizer lies in the micrometer range leading to the design of an ultracompact polarizer with degree of polarization as one and high transmittance. It has also been shown that by tailoring the radius of the defect rods, one can design a superpolarizer for the required wavelength.

Further, the polarizer action observed for this PBG polarizer using the FDTD method is in accordance with the band diagrams for the considered structure obtained from the PWE method.

However, in this letter, we have mainly focused on the $\mathrm{PhCs}$ of dielectric columns in air; similar principles may also be applied to their counterparts, that is, $\mathrm{PhCs}$, of low index material embedded in a high index background if the structure fulfills the PBG requirements.

\section{Acknowledgment}

The authors gratefully acknowledge the (i) financial support provided by All India Council of Technical Education, Government of India for the research and development project "Propagation Characteristics of Photonic Crystal Fibers and Waveguides for Telecom and Sensing Applications" and (ii) initiatives toward the establishment of "TIFAC-Center of Relevance and Excellence in Fiber Optics and Optical Communications at Delhi College of Engineering, Delhi" through "Mission REACH" program of Technology Vision-2020, Government of India.

\section{References}

1. P. R. Villeneuve and M. Piche, "Photonic band gaps in twodimensional square and hexagonal lattices," Phys. Rev. B 46, 49694972 (1992).

2. P. R. Villeneuve and M. Piche, "Photonic band gaps in twodimensional square lattices-square and circular rods," Phys. Rev. B 46, 4973-4975 (1992).

3. M. Qiu and S. He, "Large complete band gap in two-dimensional photonic crystals with elliptic air holes," Phys. Rev. B, 60, 1061010612 (1999).

4. M. Qiu, "Band gap effects in asymmetric photonic crystal slabs," Phys. Rev. B 66, 033103 (2002).

5. Y. Nagpal and R. K. Sinha, "Polarization dependent analysis of photonic crystal direction coupler," J. Microwaves and Optoelectronics 3, 39-46 (2004).

6. M. Bayindir and E. Ozbay, "Band dropping via coupled photonic crystal waveguides," Opt. Express 10, 1279-1284 (2002).

7. M. Kamp, T. Happ, S. Mahnkopf, G. Duan, S. Anand, and A. Forhel, "Semiconductor photonic crystals for optoelectronics," Physica E (Amsterdam) 21, 802-808 (2004).

8. E. A. Camargo, H. M. H. Chong, and R. M. De La Rue, "Four port coupled channel-guide device based on 2D photonic crystal structure," Photonics Nanostruct. Fundam. Appl. 2, 227-213 (2004).

9. Y. Nagpal and R. K. Sinha, "Modeling of photonic band gap waveguide couplers," Microwave Opt. Technol. Lett. 43, 47-50 (2004).

10. T. Niemi, L. H. Frandsen, K. K. Hede, A. Harporth, P. I. Borel, and M. Kristenen, "Wavelength-division demultiplexing using photonic crystal waveguides," IEEE Photonics Technol. Lett. 18, 226-228 (2006).

11. Y. Kalra and R. K. Sinha, "Design of ultra compact polarization splitter based on the complete photonic band gap," Opt. Quantum Electron. 37, 889-895 (2005).

12. L. Wu, M. Mazillu, J. F. Gallet, T. F. Krauss, A. Jugessur, and R. M. De La Rue, "Planar photonic crystal polarization splitter,"Opt. Lett. 29, 1620-1622 (2004).

13. Y. Ohtera, T. Sato, T. Kawashima, T. Tamamura, and S. Kawakami, "Photonic crystal polarization splitter," Electron. Lett. 35, 1271-1272 (1999).

14. M. Yokoyama and S. Noda, "Polarization control of two dimensional photonic crystal laser having square lattice structure,"IEEE J. Quantum Electron. 39, 1074-1080 (2003).

15. D. R. Solli, C. F. McCormick, R. Y. Chiao, and J. M. Hickmann, "Photonic crystal polarizers and polarizing beam splitters," J. Appl. Phys. 93, 9429-9431 (2003).

16. S. K. Mondal and B. J. H. Stadler, "Novel designs for integrating YIG/Air photonic crystal slab polarizers with waveguide faraday rotators," IEEE Photonics Technol. Lett. 17, 127-129 (2005). 